\title{
French cross-cultural adaptation and validation of the Quality of Life-Alzheimer's Disease scale in Nursing Homes (QOL-AD NH)
}

\author{
Christophe Cousi ${ }^{1,2^{*}} \mathbb{0}$, Valérie Igier $^{1}$ and Bruno Quintard ${ }^{2}$
}

\begin{abstract}
Background: No specific scale to measure Quality of Life in Alzheimer's Disease in Nursing Homes (QoL-AD NH) exists in French. We aimed to translate and culturally adapt the QoL-AD NH participant scale into a French version and evaluate its psychometric properties with residents in French nursing homes (EHPAD).

Methods: First, the QoL-AD NH was cross-culturally adapted into French according to guidelines. Secondly, a convenience group of residents with mild to moderate dementia answered the Folstein's test and the QoL-AD NH. They also answered the Dementia Quality of Life and the Geriatric Depression Scale to test convergent and divergent validity. Known-group validity was tested with a comparison group of residents without dementia. Exploratory Structural Equation Modeling (ESEM) was used after Exploratory Factor Analysis (EFA) to identify factors and measure invariance across age and mental state groups. Reliability (internal consistency, McDonald's omega and test-retest) were also measured.
\end{abstract}

Results: Following successful adaptation of the QoL-AD NH, 174 residents (mean age 86.6) from 7 nursing homes with mild to moderate dementia participated in the validation study. We retained a 3 -factor model of the scale after ESEM identifying: "Intra \& interpersonal environment-related QoL", "Self-functioning-related QoL" and "Perceived current health-related QoL" that were invariant across age and mental state groups. The QoL-AD NH had acceptable convergent ( $\rho$ range $0.24-0.53$ ) and divergent validity ( $\rho$ range -0.43 to -0.57 ) and good known-group validity with 33 residents without dementia $(t(205)=2.70, p=.007)$. For reliability, the results revealed very good and adequate internal consistency ( $a=0.86$ for total scale and $\geq 0.71$ for subscales). All total omega values exceeded the threshold 0.70. The hierarchical omega was 0.50 , supporting the multidimensionality of the scale. Hierarchical omega subscale values exceeded the minimal level 0.50 except for the third factor, although reliable, would deserve more items. Testretest was good with ICC $(3,1)=0.76$.

Conclusions: The QoL-AD NH French participant version has globally good reliability and validity for evaluating residents' quality of life. However, further studies must rework and confirm the factor structure, test sensitivity to change and responsiveness.

Keywords: Quality of life, Dementia, Alzheimer's disease, Nursing homes, QOL-AD

*Correspondence: christophe.cousi@univ-tlse2.fr

1 CLESCO ED 326, Centre for Studies and Research in Psychopathology and Health (CERPPS), University of Toulouse Jean-Jaurès, Toulouse, France

Full list of author information is available at the end of the article

\section{Background}

In France, nursing homes for elderly (EHPAD-Établissement d'Hébergement pour Personnes âgées Dépendantes) are accommodating increasingly older residents with chronic illnesses and major cognitive impairments. More than $70 \%$ of EHPAD residents can have major cognitive original author(s) and the source, provide a link to the Creative Commons licence, and indicate if changes were made. The images or other third party material in this article are included in the article's Creative Commons licence, unless indicated otherwise in a credit line to the material. If material is not included in the article's Creative Commons licence and your intended use is not permitted by statutory regulation or exceeds the permitted use, you will need to obtain permission directly from the copyright holder. To view a copy of this licence, visit http://creativecommons.org/licenses/by/4.0/. The Creative Commons Public Domain Dedication waiver (http://creativeco mmons.org/publicdomain/zero/1.0/) applies to the data made available in this article, unless otherwise stated in a credit line to the data. 
impairments [1-3]. Knowing that the average length of stay of a resident in a nursing home is 3.6 years [4], evaluating and improving quality of life in nursing homes is a critical societal issue. In this way, the French National Agency for the Evaluation and Quality of Social and Medico-social Establishments and Services (ANESM) has included an approach focused on Quality of life in EHPAD in its work program [5]. In strand 1 of this program, the ANESM declares (p. 7):

An EHPAD is a place where the aim is the quality of life of each resident throughout their stay, whatever their difficulties: physical dependence, loss of decision-making autonomy, or difficulties in expressing themselves.

The concept of quality of life (QoL) has been conceptualised and defined differently, making it difficult to measure. In 1994, the World Health Organization defined QoL as:

An individual's perceptions of their position in life in the context of the culture and value systems in which they live, and in relation to their goals, expectations, standards and concerns.

In the context of elderly cognitive impairment, there is emerging agreement on the meaning of QoL [6-8]. Lawton's model is the most widely used conceptualisation of QoL in Alzheimer's disease (and related disorders), including both objective and subjective factors based on four fundamental dimensions: "Psychological well-being" (e.g., positive and negative affects), "Behavioural skills" (e.g., cognitive and functional abilities), "Objective environment" (caregivers, helpers and living situation) and "Perceived QoL" [9]. According to this model, self-report would be preferable when feasible, and proxy-reports would occur when not feasible. However, a recent systematic study advocated the use of a proxy observation tool, the QUALIDEM, to assess the QoL of residents with cognitive impairments [10], although families and caregivers have been reported to undervalue the QoL of the person with cognitive impairment [11-15]. Nevertheless, despite the presence of cognitive impairment, many studies have shown that patients with Alzheimer's disease were fully able to assess their QoL [11, 16-18], even up to an advanced disease stage [12, 19-21].

Over the past twenty-five years, researchers have developed many scales to assess HRQoL specific to dementia, yet there is not yet a gold standard to measure QoL in Azheimer's disease [14, 22]. One scale, widely used in studies, is the QoL in Alzheimer's Disease scale (QoLAD 13) [23-25]. This scale is available in many European countries after cross-cultural adaptations [26-30], including France [31]. However, this scale may not be optimal for assessing QoL of residents who live in institutions as some items are specific to assess QoL at home. That is why Edelman et al. [13] slightly modified this tool to measure more precisely QoL in Alzheimer's disease in elderly institutions/nursing homes with participant and proxy versions. While some researchers have used the QoL-AD NH scale in both versions (self and proxyrated) [11-14, 32] or self-rated version only [33], others have only focused on proxy evaluation [15, 34-37] like Edelman et al. [13] who used the QoL-AD NH in a proxy evaluation with staff for the first time.

Several recent systematic reviews have emphasised weak or unexplored psychometric properties of dementia-specific QoL instruments for use in care settings, including the QoL-AD NH [10, 22, 24, 38]. Indeed, almost all studies have used the QoL-AD NH as an outcome measure but have never tested psychometric properties of the participant's version. Only two studies have examined dimensionality of the QoL-AD NH, using only 11 items out of 15 on the scale [27,39]. As a result, the QoL-AD NH scale has not yet shown real psychometric qualities, even in its original English version since there is no psychometric validation study. Finally, while there are scales that assess the HRQoL among community-dwelling older people in France [31, 40], there is, to our knowledge, no specific self-rating scale to assess residents' QoL in Alzheimer's disease in France, and more globally in the French-speaking world.

Our research aimed to provide a French cross-cultural adaptation and a first psychometric validation of the QoL-AD NH Participant version for French nursing home residents with mild to moderate Alzheimer's disease or related disorders.

\section{Methods \\ Study design}

We led a descriptive cross-sectional study in two phases. In phase 1 , the translation and cross-cultural adaptation of the QoL-AD NH (Participant version during an interview) from English into French was undertaken. In phase II, psychometric validation of the French adaptation was performed.

Ethical approval was obtained from the CERNI Ethics Committee of the University of Toulouse (number 2017-064).

\section{Phase 1: Translation and cross-cultural (transcultural) adaptation of the QoL-AD NH participant version} According to cross-cultural adaptation guidelines, a French adaptation was carried out, which involves adaptation, not just translation [41]. Several steps were necessary. 


\section{Translation (from English to French)}

First of all, two French native-speaking translators independently translated the original English version of the QoL-AD NH; one of the translators (T1) was familiar with the study's concepts and the medical environment, the other (T2) was not.

\section{Synthesis}

A synthesis of the two translations was carried out, and a consensus was reached to develop a T-12 version.

\section{Back translation (from French to English)}

Working from the T-12 version of the scale, two English mother-tongue translators who were naive to the concepts and the medical environment carried out the back translation and produced $\mathrm{B} 1$ and $\mathrm{B} 2$ versions.

\section{Expert committee review}

After the translations, an expert committee met with the four translators, the principal investigator (CC) and the two co-authors (BQ, VI); the first one was specialised in QoL, the other in gerontology. This expert committee produced a pre-final version of the scale. The role was to consolidate all the translated versions considering four aspects: (1) semantic equivalence, (2) idiomatic equivalence, (3) experiential equivalence, and (4) conceptual equivalence.

\section{Pretesting}

The aim was to individually test the comprehension of the items and instructions of the adapted interview guide as well as the understanding and clarity of the response system. Ultimately, the aim was to assess the extent to which residents with mild to moderate cognitive impairment could complete the questionnaire according to the instructions and the proposed response system before use in the psychometric validation study.

Both Rebecca Logsdon (the original author and who participated in this French adaptation) and Perry Edelman (the lead author of the English nursing home adaptation) approved our cross-cultural adaptation into French.

\section{Phase 2: Psychometric validation of the QoL-AD NH French Participant version \\ Participants}

Participants were residents of several nursing homes from the southwest of France.

They were recruited in two convenience samples according to different inclusion and exclusion criteria.

Inclusion criteria for the main sample were:

- To be at least 60 years old and a native French speaker
- To be a resident in the nursing home for more than three months

- To have a major neurocognitive disorder from mild to moderate stage

- To have a Mini-Mental State Examination (MMSE) score $\geq$ of 10

Exclusion criteria for the main sample were:

- Residents with major hearing or visual impairments despite the correction

- Residents hospitalised in the 30 days before the interview

- Residents with excessive cognitive-behavioural problems precluding answering the questions.

A comparison group of residents without cognitive impairment was also recruited. Criteria for non-cognitive impairment were confirmed by taking age and education into account in the MMSE score [42] and with the study group's first two inclusion/exclusion criteria.

The main sample size was based on the recommendations proposed by the Consensus-based Standards for the Selection of Health Measurement Instruments (COSMIN) to have at least five to ten times more participants per item of the questionnaire [43]. For this purpose and security purposes, the minimum estimated sample was 150. The sample size for the comparison group was set at a minimum of 30 participants and 25 participants for test-retest reliability (randomly extracted from the main group).

\section{Questionnaires}

A sociodemographic questionnaire indicated gender, age, education level, family situation, type of dementia, and dependency level, from GIR 6 (complete autonomy) to GIR 1 (totally dependent). This information was collected from the residents' medical records. Moreover, we noted any possible legal protection measure.

The protocol administered included the following questionnaires:

The Mini-Mental State Examination (MMSE) [44]

This scale assesses cognitive abilities, from no cognitive impairment to very severe cognitive impairment [45]. We used the GRECO version adapted and calibrated in French [42, 46]. In the absence of evidence to determine an MMSE cut-off score between mild and moderate cognitive impairment, two groups were created for comparison, taking as cut-off value 
the median of the MMSE scores [27]. Scores can range from 0 to 30 (higher scores indicating better cognitive status). MMSE scores were obtained either from the nursing home tests if run less than three months ago or as part of the study protocol. We did this to include residents in the right group according to their most current cognitive score.

The QoL in Alzheimer's Disease Nursing Home (QoL-AD NH) participant version

The QoL-AD NH is an adaptation of Logsdon et al's [23] QoL-AD scale. The QoL-AD scale was adapted to QoL-AD NH [13] to assess the QoL in Alzheimer's disease for residents in nursing homes. We used the adapted "Participant version" run directly with the resident answering questions during an interview. According to the original author of the QoL-AD 13 [23] and the QoL-AD NH [13], patients with an MMSE score $\geq 10$ can complete the questionnaire. The scale includes 15 items rating from 1 to 4 points each: 1 (Poor); 2 (Fair); 3 (Good); 4 (Excellent) and total scores ranging from 15 to 60 . Questionnaires with a maximum of two missing items were considered valid.

Dementia Quality of Life (DQoL)

The Dementia QoL (DQoL) scale was validated in English with a sample of 99 patients with dementia [16] and was adapted into French with elderly people with mild to moderate dementia [47]. This tool measures the construct of perceived QoL in Alzheimer's disease of the elderly. It consists of 29 items on a 5-level Likert scale and covers five factors: (1) "Self-esteem" (2) "Positive affect/Humor" (3) "Negative affect" (4) "Feelings of belonging" (5) "Sense of Aesthetics". A final optional item assessing the overall QoL was not used in this study. An average score was calculated for each DQoL dimension. This scale was recommended by a systematic review to assess QoL of residents with cognitive impairments [48]. So, we chose this scale to test convergent validity. The Geriatric Depression 15-item Scale (GDS-15)

The GDS-15 assesses depressive complaints and the intensity of depression in the elderly $[49,50]$. This scale validated in French [51] was well suited to older people without cognitive impairment and older people with mild to moderate cognitive impairment [52]. GDS-15 is rated yes/no. The minimum score is 0 , and the maximum is 15 . The higher the score, the more depressive symptoms and intensity are present. We chose this scale to test divergent validity.

\section{Data collection}

Twenty-one nursing homes (EHPAD) in France's southern Aquitaine region were initially contacted by phone to interview nursing home residents. The initial contact was often made with the psychologist of the EHPAD, who informed the nursing home director of the study and requested their participation. We clearly explained that the study only evaluated the perceived (and therefore subjective) resident's QoL and not the care or facility's quality.

When the structures agreed, nursing home management initially informed residents and families that research would be conducted within the facility on the QoL of EHPAD residents. Taking into account potential participant vulnerability, we obtained the agreement of the curator when the resident was under legal protection. The research protocol was conducted on the study site exclusively by the same person (CC) who had good experience with residents and could give them the necessary time and support before, during and after the research protocol.

The recruitment of residents was first carried out with the help of the health care teams. They drew up an initial list of residents likely to be able and willing to respond to the questionnaire and according to the inclusion/exclusion criteria. The residents' medical and administrative records were consulted to confirm the caregivers' indications for Alzheimer disease (or related disorders) and collect the sociodemographic information. The data was then made anonymous.

Residents were informed of the study and individually invited to participate of their own free will in the research by asking for their agreement and when and at what time they wished to answer the questions. Before the interview, a simplified and large-print information and consent form was presented to residents explaining the aim of the research, the approximate duration, and data confidentiality and anonymity for aggregate results for scientific publication with a poster feedback of the simplified results that would be offered to them. They were also informed that they could stop the interview at any time and stop it permanently or resume it later if they so wished. Finally, we obtained their written consent signed in duplicate, one copy of which was given to the resident. The interview took place in their rooms.

The data collection was conducted from January to February 2020 before the Covid-19 pandemic and subsequent lockdowns.

\section{Statistical analysis}

Descriptive statistics Descriptive statistics were presented as percentages, mean $( \pm S D) /$ Range, age, type of dementia, MMSE score, gender, marital status, educational status, and GIR (level de dependency). 
Acceptability At first, the QoL-AD NH acceptability was assessed by the number of refusals and current dropouts and then by the percentage of missing data (rate of completed items) [53]. Floor and ceiling effect were analysed with a permissible limit of 15\% [43]. Lastly, the administration time was calculated.

Normality test Variables were assessed for univariate and multivariate normality. For skewness, if data were greater than +1 or lower than -1 and for kurtosis data were greater than +1 , then data distribution essentially deviated from a normal distribution [54]. Multivariate normality for QoL-AD NH was evaluated using the Mardia test with a critical ratio below 8.00 , indicating multivariate normality [55]. To identify univariate outliers, the variables were standardised. The cases with values exceeding 3.29 were considered outliers [56]. To evaluate multivariate outliers, the values of "Mahalanobis distance" were generated. The presence of possible outliers moving away from "centroids" and whose Mahalanobis $D^{2}$ values were lower than 0.001 were considered multivariate outliers [56].

Factor analysis We followed a two-step approach based on exploratory structural equation modeling (ESEM) [57]. ESEM can be seen as a compromise between the flexibility of EFA and the rigour of SEM [58]. While its application lies mainly in confirmatory research, ESEM has been used when factor structures were not yet well established $[59,60]$. First, exploratory factor analysis (EFA) was conducted to determine factor loadings. The resulting loading matrix was then used to formulate a structural equation model (SEM), allowing for a more detailed model fit assessment.

Parallel Analysis (PA) was used to determine the optimal factors to extract [61]. We simulated 1000 random datasets. In addition to the model proposed by the parallel analysis, we fitted different EFA models that assumed one, two, or three factors. Fit indices of these models were compared in the ESEM step.

Exploratory factor analyses were conducted using maximum likelihood estimation and goemin rotation and an epsilon value of 0.50 [57, 62]. Geomin is an oblique rotation method, so the extracted factors could be correlated.

Once all EFA models were fit, ESEM was performed using their implied loading matrices. As mentioned above, ESEM was used to provide further guidance for selecting an adequate factor structure [63]. As cut-off criteria for good model fit, we mainly used the values provided by $\mathrm{Hu}$ and Bentler [64]: a model will be accepted if the Tucker-Lewis Index (TLI) surpasses 0.94 and the standardised root mean square residual (SRMR) is below 0.09 .
Three criteria were taken into account to choose a model from our exploratory analysis: the interpretability of the factors, the model's simplicity, and the fit indices.

Measurement invariance Measurement invariance was tested between three age groups: persons of 60 to 84 years of age $(n=50), 84$ to 90 years $(n=60)$, and 90 to 100 years $(n=64)$. Measurement invariance was also tested between two groups by cognitive level (MMSE) with similar sizes: residents with MMSE scores below the median value (19), $n=90$, and residents with MMSE scores above than the median value, $n=84$.

We estimated a series of models with increasing equality constraints. The decline in model fit could easily be examined. If the difference in model fit after increasing constraints decreased too much, it could be concluded that measurement invariance did not hold. Following the cut-off scores proposed by Cheung and Rensvold [65], measurement invariance does not hold if the CFI drops by more than 0.01 .

Convergent and divergent validity Convergent validity was assessed using Spearman's Rhô correlation comparing the QoL-AD NH with the DQoL. Divergent validity was also assessed using Spearman's Rhô correlation, comparing the QoL-AD NH with the GDS-15. We retained the following values: weak $(\rho<0.25)$, moderate $(0.25<\rho<0.50)$, good $(0.50<\rho<0.75)$, and excellent $(\rho>0.75)$ [66]. We formulated four hypotheses inferred from the literature: 1) The total score of the QoL-AD NH would correlate positively and moderately with the scores of the four positive dimensions of the DQoL scale. 2) (If multifactor solution) The scores of the retained factors of the QoL-AD NH would correlate positively and moderately with the scores of the four positive dimensions of the DQoL scale. 3) The total score of the QoL-AD NH would correlate negatively and well with the score of the GDS15. 4) (If multifactor solution) The scores of the retained factors of the QoL-AD NH would correlate negatively and well/moderately with the score of the GDS-15. Convergent and divergent validity were confirmed if at least $75 \%$ of the results corresponded with the hypotheses [43].

Known-group validity The objective was to discriminate across groups known to differ theoretically [67]: residents with cognitive impairment and those without. Knowngroup validity was evaluated using an Independent Samples t-Test to test QoL scores between two groups. Secondly, a one-way ANOVA followed by a post hoc Bonferroni test to assess QoL scores between three groups (residents without cognitive impairment, mild cognitive impairment and moderate cognitive impairment). Based on the literature, we hypothesised that the group with 
dementia would have significantly lower scores of QoL than the residents without cognitive impairment. We also assumed that there would be a significant difference in QoL scores between the three groups (without, mild and moderate dementia). Known-group validity was confirmed if at least $75 \%$ of the results corresponded with the hypotheses [43].

Reliability analysis Internal consistency. Internal consistency was assessed with Cronbach's alpha. We used Kline's Cut-offs with coefficients around 0.90 considered "excellent," values around 0.80 as "very good," and values from 0.70 as "adequate" [68].

McDonald's total omega, hierarchical omega, and hierarchical subscales. In multidimensionality, Cronbach's alpha values often underestimate the reliability of scales [69]. Coefficient omega [70] allows to model sources of variance at general and multidimensional levels and is the more suitable estimate of reliability in the case of hypothesised multidimensionality [69, 71-73]. The bifactor model with the general QoL factor and multidimensional factor (factors determined by EFA/ESEM) was used to estimate omega values $[69,73,74]$. Three different omega values (total, hierarchical, and hierarchical subscales) were estimated to examine the factor solution received at EFA/ESEM stage. Total omega was estimated for the overall scale and three subscales. Total omega values above 0.70 indicate an acceptable level of composite reliability [75]. Hierarchical omega shows the proportion of variance attributable to a general factor [69]. There is no commonly accepted cut-off for hierarchical omega. A value above 0.50 is recommended as a low-level hierarchical omega, indicating that the scale is a valid instrument to measure a general factor [71]. The hierarchical omega subscale indicates the proportion of subscale scores variance explained by the multidimensional (subscale) factor after controlling for the effect of the general factor [76]. The value of the hierarchical omega subscale above 0.50 is considered enough for the reliability of a subscale [71].

Test-retest analysis. Test-retest reliability was assessed in a two-week interval in a group of twenty-five residents with mild to moderate cognitive impairment. In order to compare results with previous studies, we used both Pearson's correlation and the Intraclass Correlation Coefficient of type ICC $(3,1)$ with a 2-way mixed-effects model, single measurement, unique rater and absolute agreement. It was based on the following values: less than 0.50 (poor), between 0.50 and 0.75 (moderate), between 0.75 and 0.90 (good), and above 0.90 (excellent) [77].

All statistical analyses were done using $\mathrm{R}$ version 4.1.0 (2021-05-18) - "Camp Pontanezen", Brest, France [78] and the integrated development environment RStudio 1.4.1103 [79].

\section{Results \\ Phase 1: Translation and cross-cultural adaptation of the QoL-AD NH French participant version}

During the cross-cultural adaptation phase with the expert committee, some items had to be adapted, including item 09 "Personality overall" whose translation into "Self-image" was maintained (similar to the validated 13-item French version) [31]. Also, item 12 "Life overall" was adapted to "Current life in general" to clearly distinguish the desired meaning of current quality of life from life satisfaction. The supplementary document containing the instructions and scale questions for each item was also adapted. A pre-final version was developed and then pre-tested with twelve residents suffering from mild to moderate cognitive impairment who were not part of the sample for psychometric validation. This pre-final version proved to be well adapted and required only a few explanations for the item 09 "Self-image" and the item 15 "Ability to make choices in one's life" with a slight rewording of the phrasing of the questions in the interview guide.

\section{Phase 2: Psychometric properties of the French QoL-AD NH French Participant version Descriptive statistics}

Of the twenty-one nursing homes contacted, seven agreed to participate, i.e. 1/3 of them. Four were private structures, and three were associations. The size and capacity of these structures varied from 35 to 90 residents. A total of one hundred and seventy-four residents with mild to moderate cognitive impairment participated in the research (Table 1) and a comparison group of thirty-three residents without cognitive impairment joined the study only to test known-group validity. Both groups had equivalent socio-demographic characteristics but the comparison group did not suffer from any cognitive impairment, with an average MMSE score of 26 $( \pm 2.6)$ on a scale of 24 to 30 .

\section{Acceptability}

All invited participants accepted to participate and completed the protocol. The completion rate was $99.6 \%$ for the QoL-AD NH. No floor $(0 \%)$ or ceiling effect $(0 \%)$ was identified for the QoL-AD NH. With the presentation of the scale and the instructions, the average time taken to complete this questionnaire was $11 \mathrm{~min}$. 
Table 1 Description of nursing home residents with Alzheimer's disease or related disorders $(n=174)$

\begin{tabular}{|c|c|c|c|}
\hline & $N$ & $\%$ & Mean $( \pm$ SD)/Range \\
\hline Age in years & 174 & 100.0 & $86.6( \pm 7.6) / 60-100$ \\
\hline $60-69$ & 9 & 5.2 & \\
\hline 70-79 & 16 & 9.2 & \\
\hline $80-89$ & 75 & 43.1 & \\
\hline $90-100$ & 74 & 42.5 & \\
\hline Type of probable dementia & 174 & 100.0 & \\
\hline Alzheimer's disease & 138 & 79.3 & \\
\hline Mixed dementia & 24 & 13.8 & \\
\hline Vascular dementia & 12 & 6.9 & \\
\hline \multicolumn{4}{|l|}{ MMSE score } \\
\hline Total score & 174 & 100.0 & $18.8( \pm 5.1) / 10-26$ \\
\hline$<19$ & 78 & 81.6 & $13.9( \pm 6.6) / 10-18$ \\
\hline$\geq 19$ & 96 & 18.4 & $22.7( \pm 2.3) / 19-26$ \\
\hline \multicolumn{4}{|l|}{ Gender } \\
\hline Female & 142 & 81.6 & \\
\hline Male & 32 & 18.4 & \\
\hline \multicolumn{4}{|l|}{ Marital status } \\
\hline Single & 54 & 31.1 & \\
\hline Married & 11 & 6.3 & \\
\hline Divorced & 4 & 2.3 & \\
\hline Widowed & 105 & 60.3 & \\
\hline \multicolumn{4}{|l|}{ Educational status } \\
\hline No qualification & 46 & 26.4 & \\
\hline Primary diploma & 80 & 46.0 & \\
\hline Secondary & 29 & 16.7 & \\
\hline Higher education & 19 & 10.9 & \\
\hline \multicolumn{4}{|l|}{$G I R^{*}$} \\
\hline GIR 1 & 1 & 0.6 & \\
\hline GIR 2 & 39 & 22.4 & \\
\hline GIR 3 & 41 & 23.6 & \\
\hline GIR 4 & 72 & 41.3 & \\
\hline GIR 5 & 16 & 9.2 & \\
\hline GIR 6 & 5 & 2.9 & \\
\hline
\end{tabular}

" GIR level of dependency from GIR 1 (the most dependant) to GIR 6 (the least dependant)

\section{Normality test}

The Skewness of two items (item 4 \& item 6) were just above -1 . As a result, these variables were not normally distributed because there were negatively skewed. A correction $\log 10(\max (x+1)-x)$ was applied to try to correct negatively skewed data with success. If the QoL-AD $\mathrm{NH}$ continuous variables were normally distributed, continuous variables from the GDS-15 and the dimension "Feeling of belonging" of the DQoL were not. The critical ratio of the Mardia test was 7.84, so there was multivariate normality [55]. There was no univariate or multivariate outlier [56].

\section{Factor structure}

Item 12 "Current life in general" was not included in the factor analysis because it was the general item of the scale and was identical to the Dementia Quality of Life Scale item that was not part of the factor structure but as an item evaluating and generally summarising the perceived QoL [16, 39]. Also, as it is a QoL in Alzheimer's disease scale, only the 174 residents with Alzheimer's disease or related disorders from mild to moderated cognitive impairment were included in factor analysis.

Parallel Analysis (PA) A parallel analysis was conducted. Finally, four factors were suggested for exploratory factor analysis.

Exploratory Factor Analysis (EFA) The KaiserMeyer-Olkin measure of sampling adequacy (KMO) was 0.84 and Bartlett's test for sphericity was significant $\left(\chi^{2}(91)=699.34, p<0.001\right)$, so we proceeded with the factor analysis. Four EFA models (models 1 to 4 ) with one to four factors were examined. Their goodness-of-fit statistics are displayed in Table 2.

Exploratory Structural Equation Modeling (ESEM) The results showed that the one-factor and two-factor models did not fit. Only the three-factor and four-factor models had good fit indices (Table 2).

The four-factor model adjusted too much to the data indicating probable overfitting. Moreover, two of the four factors were based on only two items, and one of the other factors was difficult to interpret (Additional file 4). On the contrary, the three-factor model was easy interpretable, simple and a good fit to data. As a result, this

Table 2 Comparison of the four models by their fit indices

\begin{tabular}{llllllll}
\hline & $\boldsymbol{x}^{\mathbf{2}}$ & $\boldsymbol{d f}$ & $\boldsymbol{p}$ & CFI & RMSEA & TLI & SRMR \\
\hline One factor & 193.52 & 90 & 0.000 & 0.821 & 0.085 & 0.819 & 0.082 \\
Two factors & 143.13 & 88 & 0.000 & 0.905 & 0.062 & 0.902 & 0.068 \\
Three factors & 88.50 & 85 & 0.390 & 0.995 & 0.015 & 0.994 & 0.051 \\
Four factors & 56.60 & 81 & 0.982 & 1.000 & 0.000 & 1.047 & 0.042 \\
\hline
\end{tabular}

$\mathrm{X}^{2}$, chi-square; df, degrees of freedom; CFI, comparative fit index; RMSEA, root mean square error of approximation; TLI, Tucker-Lewis index; SRMR, standardised root mean square residual 
three-dimensional model was chosen. Standardised factor loadings of this model are summarised in Table 3.

The three factors were interpreted as follows: Factor 1: "Intrapersonal \& interpersonal environment-related QoL"; Factor 2: "Self-functioning-related QoL"; Factor 3: "Perceived current health-related QoL" (Table 3).

\section{Measurement invariance (MI) of the selected model}

Measurement invariance analysis by age group showed no significant drop in model fit. We observed a comparative fit index (CFI) of 0.889 for the configural invariance model which remained constant in the metric invariance model. Finally, a drop of 0.003 was observed in the scalar invariance model [65]. It was concluded that the threefactor model was measurement invariant by age group (Table 4).

Regarding measurement invariance by mental state, we did not observe decreasing CFA and increasing RMSEA when comparing models. Also, fit CFA and RMSEA values indicated an acceptable fit for configural and constrained models [65]. Thus, the 3-factor model demonstrated measurement invariance across groups with lower and higher MMSE scores (Table 5).

Table 3 Standardised factor loadings, eigenvalues, factor intercorrelations, and variance explained for 3-factor model

\begin{tabular}{llll}
\hline Item & Factor $\mathbf{1}$ & Factor $\mathbf{2}$ & Factor 3 \\
\hline Physical health & -0.02 & 0.02 & $\mathbf{0 . 8 9}$ \\
Vitality & 0.09 & 0.10 & $\mathbf{0 . 5 3}$ \\
Moral/mood & $\mathbf{0 . 5 0}$ & -0.03 & 0.27 \\
Living environment & $\mathbf{0 . 6 3}$ & 0.07 & -0.09 \\
Memory & 0.12 & $\mathbf{0 . 3 5}$ & 0.01 \\
Relationship with family & $\mathbf{0 . 4 4}$ & -0.07 & 0.00 \\
Relationship with staff & $\mathbf{0 . 6 3}$ & -0.17 & 0.08 \\
Relationship with friends & $\mathbf{0 . 4 6}$ & 0.18 & -0.07 \\
Self-image & $\mathbf{0 . 3 8}$ & 0.16 & 0.16 \\
Keep busy & 0.02 & $\mathbf{0 . 6 9}$ & 0.08 \\
Do things for pleasure & -0.04 & $\mathbf{0 . 6 8}$ & 0.12 \\
Self-care & $\mathbf{0 . 3 6}$ & 0.13 & 0.15 \\
Live with others & $\mathbf{0 . 4 3}$ & 0.21 & -0.01 \\
Make choices & 0.19 & $\mathbf{0 . 5 8}$ & -0.07 \\
Eigenvalues & 4.79 & 1.36 & 1.20 \\
Factor intercorrelations & & & \\
Factor 1 & - & & \\
Factor 2 & 0.47 & - & - \\
Factor 3 & 0.38 & 0.41 & $12 \%$ \\
Variance explained & $16 \%$ & $13 \%$ & \\
\hline
\end{tabular}

Bold numbers represent the most appropriate items corresponding to each factor, as indicated by their maximum factor loading
Table 4 Measurement invariance across age groups

\begin{tabular}{lll}
\hline & cfi.robust & rmsea.robust \\
\hline Configural & 0.889 & 0.073 \\
Metric invariance & 0.889 & 0.073 \\
Scalar invariance & 0.885 & 0.071 \\
\hline
\end{tabular}

\section{Convergent validity}

The results showed that the QoL-AD NH Total score correlated positively and well/moderately with the four positive dimensions of the DQoL, "Sense of aesthetics" $(\rho=0.44, p<0.01)$, "Positive affect" $(\rho=0.51, p<0.01)$, "Feeling of belonging" $(\rho=0.46, p<0.01)$, "Self-esteem" $(\rho=0.53, p<0.01)$ thus confirming the hypothesis. Moreover, the three factors of the QoL-AD NH correlated positively with the four positive dimensions of the DQoL: Factor 1 score "Intrapersonal \& interpersonal environment-related QoL" correlated positively and moderately/ well with the four positive dimensions of the DQoL, "Sense of aesthetics" $(\rho=0.42, p<0.01)$, "Positive affect" ( $\rho=0.52, \quad p<0.01)$, "Feeling of belonging" $(\rho=0.49$, $p<0.01)$, "Self-esteem" $(\rho=0.43, p<0.01)$ confirming the hypothesis; Factor 2 score "Self-functioning-related QoL" correlated positively and moderately with the four positive dimensions of the DQoL, "Sense of aesthetics" $(\rho=0.38, p<0.01)$, "Positive affect" $(\rho=0.39, p<0.01)$, "Feeling of belonging" $(\rho=0.30, p<0.01)$, "Self-esteem" $(\rho=0.43, p<0.01)$ confirming the hypothesis; Factor 3 score "Perceived current health-related QoL" correlated positively and moderately/weakly with the four positive dimensions of the DQoL, "Sense of aesthetics" $(\rho=0.25$, $p<0.05)$, "Positive affect" $(\rho=0.22, p<0.05)$, "Feeling of belonging" $(\rho=0.24, p<0.05)$, "Self-esteem" $(\rho=0.42$, $p<0.01$ ) partially confirming the hypothesis (Additional file 1: Table S1). These results revealed acceptable convergent validity [43].

\section{Divergent validity}

The QoL-AD NH total score was highly and negatively correlated with the GDS-15 scale score $(p=-0.57, p<0.01)$, confirming the hypothesis. Moreover, the GDS-15 score correlated negatively and moderately with the three factors of the QoL-AD NH: with Factor 1 score "Intrapersonal

Table 5 Measurement invariance across the mental status groups

\begin{tabular}{lll}
\hline & cfi.robust & rmsea.robust \\
\hline Configural & 0.922 & 0.059 \\
Metric invariance & 0.922 & 0.059 \\
Scalar invariance & 0.928 & 0.055 \\
\hline
\end{tabular}


\& interpersonal environment-related QoL" $(\rho=-0.48$, $p<0.01$ ), with Factor 2 score "Self-functioning-related QoL" $(\rho=-0.45, p<0.01)$ and with Factor 3 score "Perceived current health-related QoL" $(\rho=-0.43, p<0.01)$ partially confirming the hypothesis (Additional file 1: Table S1). These results revealed acceptable divergent validity [43].

\section{Known-group validity}

First of all, we compared two groups: residents without cognitive impairment $(n=33)$ and the whole group of residents with cognitive impairment $(n=174)$. As predicted, QoL of the residents with cognitive impairment was significantly lower $(37.4 \pm 6.2)$ than the QoL of residents without cognitive impairment (40.5 \pm 5.2$), t(205)=2.70$, $p=0.007$, thus confirming the hypothesis. Secondly, we compared the three groups of residents' QoL: residents with mild dementia $(n=96)$, moderate dementia $(n=78)$ and without cognitive impairment $(n=33)$. The results revealed a significant difference between groups determined by the one-way ANOVA, $F(2,204)=4.871$, $p=0.009$. A Bonferroni post hoc test showed that the residents' QoL with moderate cognitive impairment was significantly lower $(36.7 \pm 6.2, p=0.006)$ than the QoL of residents without cognitive impairment $(40.5 \pm 5.2)$. However, there was no significant difference between the QoL of residents with moderate cognitive impairment and the residents with mild cognitive impairment $(p=0.379)$ and between the residents with mild cognitive impairment and residents without cognitive impairment $(p=0.129)$. As a result, the hypothesis was partly confirmed. Globally, these results confirmed good known-group validity [43]. Finally, we resumed means and standard deviations for each group's continuous variables (Additional file 2) and each QoL-AD NH scale item (Additional file 3).

\section{Reliability analysis}

Internal consistency Cronbach's alpha for all 15 items of the QoL-AD NH was $\alpha=0.86$. So, the internal consistency of the total scale was very good [68]. Once the three-factor model was selected after factor analysis and ESEM, we measured internal consistency for the subscales (Table 6). The results showed that the Cronbach's alpha was very good for Factor 1 ("Intrapersonal \& interpersonal environment-related QoL", $\alpha=0.77)$, adequate for Factor 2 and Factor 3 ("Self-functioning-related QoL", $\alpha=0.72$; "Perceived current health-related QoL", $\alpha=0.71$ ) [68].

McDonald's total omega, hierarchical omega, and hierarchical subscales (Table 6) In the 3-factor QoL-AD NH scale, total omega values were high for both the total scale and subscale scores: 0.91 (Total scale), 0.89 ("Intrapersonal \& interpersonal environment-related QoL"), 0.84 ("Self-
Table 6 Cronbach's alpha and McDonald's total omega, hierarchical omega and hierarchical subscales for the three-factor model

\begin{tabular}{lccc}
\hline & $\boldsymbol{a}$ & $\boldsymbol{\omega}_{\mathbf{s}}(\boldsymbol{\omega})$ & $\boldsymbol{\omega}_{\mathrm{hs}}\left(\boldsymbol{\omega}_{\mathrm{h}}\right)$ \\
\hline F1 "Intraperso. \& interperso. environment-related & 0.77 & 0.89 & 0.60 \\
QoL" & & & \\
F2 "Self-functioning-related QoL" & 0.72 & 0.84 & 0.58 \\
F3 "Perceived current health-related QoL" & 0.71 & 0.74 & 0.34 \\
QoL total scale & 0.86 & $(0.91)$ & $(0.50)$
\end{tabular}

$a=$ alpha, $\omega_{s}(\omega)-\omega_{s}=$ total omega for subscales and total $\omega$ for total scale, $\omega_{\mathrm{hs}}=$ omega hierarchical for subscales, $\omega_{\mathrm{h}}=$ omega hierarchical for general factor (general factor is a latent factor of $\mathrm{QoL}$ )

functioning-related QoL"), and 0.74 ("Perceived current health-related QoL"). All total omega values exceeded the threshold 0.70 [75], supporting composite reliability of the scale in general and each of three subscales. The hierarchical omega was 0.50 . When compared to the total omega value of 0.91 , it is clear that the explained variance in the total QoL scores is attributable in approximately equal proportions to the general QoL factor (around 55\%) and multidimensionality factor (around 45\%). It indicates that raw QoL scores were essentially affected by the subscale factor supporting the multidimensionality of the QoL scale. Omega hierarchical subscale values exceeded the minimal level 0.50 [71] for "Intrapersonal \& interpersonal environment-related QoL" (0.60) and "Selffunctioning-related QoL" (0.58) subscales, indicating that the majority of subscale score variance was attributable to the multidimensional factor even after the removing effect of the general factor. The multidimensional factor contributed $67 \%$ and $69 \%$ in the total explained variance of subscale scores, indicating that the majority of reliable variance of these subscale scores was independent of the general factor. Hierarchical omega for the third factor "Perceived current health-related QoL" was 0.34, that was lower than the level 0.50. However, comparing this score with the total omega score of this subscale, 0.74 , it indicates that the subscale factor contributed significantly (46\%) to the total explained variance of subscale scores after controlling for the general factor.

Test-retest analysis Finally, test-retest reliability was calculated by the Pearson's correlation coefficient and the ICC $(3,1)$. The results revealed good test-retest reliability at 15-day intervals, with $r(23)=0.89, p=0.01$, and ICC $(3,1)=0.76, p=0.001[77]$.

\section{Discussion}

We aimed to provide a cross-cultural adaptation in French and the first psychometric validation of the QoL in Alzheimer's Disease Nursing Home Scale-Participant 
version. To our knowledge, there was no specific scale to assess QoL in Alzheimer's disease for French-speaking residents in nursing homes. Moreover, the QoL-AD $\mathrm{NH}$ had not been thoroughly analysed, even in its original English language. Our global results show that after the cross-cultural process and study validation, the French adaptation of the QoL-AD NH Participant version has globally good psychometric properties with a three-dimensional factor structure (invariant across age and mental status groups), acceptable convergent and divergent validity, good known-group validity, adequate internal consistency/omega values and good test-retest reliability.

\section{Interpretation of the results and comparison with the literature}

Since the psychometric qualities of QoL-AD NH have not been widely explored, we did not have sufficient theoretical elements to perform a Confirmatory Factor Analysis (CFA). To analyse the factor structure, we used both EFA and ESEM. The use of the ESEM combined the advantages of EFA and CFA and allowed us to select the most appropriate model while remaining in an exploratory design.

We finally retained the three-factor model, which was, at the same time, interpretable, simple and a good fit to data: Factor 1 "Intrapersonal \& interpersonal environment-related QoL" (8 items); Factor 2 "Self-functioningrelated QoL" (4 items); and Factor 3 "Perceived current health-related QoL" (2 items). These three factors seem to be representative of and pertinent to the resident's QoL in Alzheimer's disease in nursing homes. Indeed, for Factor 1 , relationships are essential for nursing home residents, especially with their families [80-82]. The more objective living environment also plays a vital role in residents' QoL with cognitive disorders, mainly when adapted to their disability and facilitates their taking temporospatial bearings $[9,81,83]$. About the "Self-functioning-related QoL" factor, it is very relevant as the resident can feel that he or she is still able to make choices, that he or she is given a choice, especially in daily activities [80, 82, 84]; this is what the person-centred care and the Montessori approach applied to the elderly advocate [85-87]. Finally, it is not surprising to find a perceived health factor in a health-related quality of life scale that usually contains physical and mental health measures with other elements not directly related to health $[53,88]$. This factor is based on only two items. However, some health-related quality of life scales can have 2-item factors [89-91], and we discuss their reliability further. Perceived health has always been important, regardless of the person's age and level of cognitive impairment [80, 92]. Moreover, the phenomenon of anosognosia may allow some residents to be not fully aware of their state of health; this may be a protector as they often report having good physical health/vitality and good QoL [93-95].

Our results contrast to the initial Edelman's study that showed a unique factor structure [13]. Indeed, in our study, the three-factor solution supports the multidimensionality of the concept of QoL in Alzheimer's disease as evoked by Lawton's model, including both objective and subjective factors [9]. Our findings also seem consistent with the Spanish validation led in day centres containing 11 of the 15 items of the QoL-AD NH, which revealed the same three-factor structure in the EFA step [27]. Nevertheless, after CFA, they decided to retain a twofactor solution even if the three-factor model fitted well [96]. Moreover, as in the Spanish study, we did not find the "Psychological well-being" factor evoked in a few validation studies of the QoL-AD 13 [97, 98]. However, our results are consistent with an Australian study conducted in long-term care facilities, revealing an identical three-factor structure [39]. This study aimed to extract a new classification system for economic evaluation from the QoL-AD NH factor structure. Based on 11 of the 15 items in the QoL-AD NH, an EFA and then a CFA confirmed a three-factor model.

Our factor analysis contained 14 of the 15 items due to excluding the global QoL item (item 12), as other studies have done [16, 39]. However, a few items loaded a bit low but were acceptable for an EFA and ESEM as they loaded at least 0.35 , in agreement with previous studies and a part of the literature admitting a threshold of 0.30 or 0.32 $[39,56,99,100]$. Moreover, there was no cross-loading because the difference between the highest loading and the second-highest loading for all items was greater than 0.20 [101]. For example, item 05 "Memory", was identified in the literature as a low loading item [23, 27, 39, 97]. However, this item is essential to the scale because it is specific to Alzheimer's disease (or related disorders) and probably more critical for a proxy evaluation of anosognosia. In that sense, we wanted to keep all the items on the one hand because the factorial results were only valid for our exploratory sample. Conversely, further studies are to be carried out to validate the hetero-evaluation version and compare the two versions. Indeed, in its original version, the author advocates calculating a composite score that would consist of $2 / 3$ of the score on the resident's evaluation and $1 / 3$ on the hetero-evaluation. In addition, as discussed below in the clinical recommendations, the individual consideration of items to assess change in the QoL would be of clinical significance and should be taken into account.

When we examined convergent validity, we found significant positive and moderate correlations between the QoL-AD NH and the DQoL. This may suggest that the 
QoL-AD NH captures further information, which is not included by the DQoL that is not specific to older people who live in a nursing home. However, the literature consistently showed significant but moderate positive correlations (i.e. around 0.30-0.63) between the QoL-AD 13 (from which QoL-AD NH derives) total score and the dimensions of the DQoL [29, 32, 102]. Also, when we examined the correlations between the three extracted factors from the QoL-AD NH and the four positive factors of the DQoL (Additional file 1), we found that the weakest correlations between the two scales lay between the "Perceived current health-related QoL" factor of the QoL-AD NH and the different factors of the DQoL. The perceived health factor that is based on 2 items may affect the correlation. However, this may be because the DQoL scale is considered as a needs assessment tool rather than an HRQoL tool [48]. The question arises as to whether the QoL-AD NH itself can be regarded as a purely HRQoL scale [39] when it originally seemed to inherit Lawton's model. While the QoL-AD was also classified as a PatientReported Outcome Measures (PROMs), the vast majority of the tools used in studies were proxy scales [103]. Concerning divergent validity, the factor "Perceived current health-related QoL" is the factor that correlates least with depression, with, even so, a moderately strong correlation. Our findings are similar to previous studies; there is a negative and significant relationship between QoL measured by the QoL-AD and depression [23, 26, 30]. Only one German study reports no relationship between QoL and depression [29]. However, many studies have shown that depression was one of QoL's strongest predictors in Alzheimer's disease [21, 25, 33, 36]. We concluded that the convergent and divergent validities were globally aligned with the other studies and acceptable.

The results of known-group validity showed that the group of residents with cognitive impairment had significantly lower QoL scores than the residents without cognitive impairment. These results agree well with existing studies $[26,104]$. However, taking into account the three groups of residents (without, mild and moderate cognitive impairment), the results showed that there was a significative difference in the QoL only between the group of residents without cognitive impairment and the group with moderate cognitive impairment, and therefore not with the group with mild cognitive impairment. This result may be due to the MMSE cut-off utilised to differentiate between groups, as we discuss further.

The question of whether the QoL-AD NH scale can also be used to assess QoL for residents without cognitive disorders is relevant. One study revealed the factor structure and measurement invariance of the QoL-AD 13 with a non-cognitive impairment community-dwelling sample [97]. This study showed a three-factor solution, including
"Physical well-being", "Social well-being" and "Psychological well-being". Our study did not extract the factor "Psychological well-being" and we had only 9 common items in the scale; it is difficult to compare the results since it is not quite the same scale nor the same sample. However, we think the QoL-AD-NH could be used to assess QoL of residents without cognitive impairment, even if elements are necessarily missing in the QoL, such as spirituality that can be important for residents who do not have cognitive disorders [10, 48].

Lawton's model, the most widely used theoretical model for conceptualising QoL in Alzheimer's disease, is not specific to Alzheimer's QoL for people living in an institution but more generally to QoL in Alzheimer's disease. However, this model takes into account the objective environment, such as the living environment. In that sense, the institutional living environment in the EHPAD nursing home probably plays an essential role in the residents' QoL, beyond purely subjective factors. In our study, item 04 "Living environment" was the strongest explanatory component of factor 1 which itself was the factor explaining most of the variance of QoL-AD NH. While Lawton's model is still the most important conceptualisation of QoL in Alzheimer's disease, it would be appropriate to develop a more specific theoretical and conceptual framework of QoL in Alzheimer's disease in institutions, mainly in nursing homes.

Regarding the reliability of the QoL-AD NH, internal consistency was very good for the total scale and one subscale, adequate for the two others. Test-retest revealed a good ICC. These results are in line with other studies [13, 26, 27, 31]. Going further than Cronbach's alpha by testing all McDonald's omega values, the global results confirmed the reliability of the subscales. Testing McDonald's hierarchical omega subscales, two subscales ("Intrapersonal \& interpersonal environment-related QoL" and "Self-functioning-related QoL") showed good reliability, contributing to subscale score variance irrespective of the general QoL factor (latent factor) influence. The third subscale "Perceived current health-related QoL", had a less independent individual effect on subscale score variance than the general QoL factor ( $46 \%$ vs $64 \%$ ). However, this effect is essential to consider that "Perceived current health-related QoL" is a reliable measure of the corresponding dimension of QoL. On the other hand, it indicates that this subscale requires future improvements, perhaps more items to increase reliability and, at the same time, validity.

\section{Strengths and limitations}

In our study, the number of participants was more extensive than similar validation studies $[26,27,29$, 96]. Moreover, our psychometric validation went further than just EFA, using the ESEM method that combines the strengths of EFA and CFA while remaining an 
exploratory process. We also went beyond Cronbach's alpha by testing all MacDonald's omega coefficients, including the omega hierarchical subscale. To our knowledge, this study is the first to examine the full validity and reliability of the QoL-AD NH, as the original version was never validated psychometrically. This study is also the first step towards the French adaptation and validation of a self-report scale that measures the QoL of nursing home residents with mild to moderate cognitive impairment.

Some study limitations should be acknowledged. First of all, we used EFA and ESEM on the same dataset; there may be a potential danger of overfitting [105]. However, we used ESEM to provide further guidance for selecting an adequate factor structure by staying in an exploratory design. Confirmatory studies will need to be conducted on another dataset. Secondly, it was difficult to determine a cut-off between mild and moderate cognitive impairment. The use of Folstein's MMSE score as a criterion of categorisation between mild and cognitive impairment could have been combined with the Clinical Dementia Rate (CDR) scale [38] or the Global Deterioration Scale to refine the level of severity and cut-off in dementia. However, these scales were not validated in French. Thirdly, the measurement invariance results across age and mental state should be interpreted with caution as the sample size to test measurement invariance was small. This is the reason why we did not use measurement invariance as a criterion for selecting the factor model. Other studies could use measurement invariance as a criterion for selecting a factor model in ESEM if they have a sufficiently large number of participants. Lastly, Factor 3 "Perceived current health-related QoL" was composed of only two items and, although valid and reliable, could be reworked by adding a few items that could improve it.

\section{Future research and clinical recommendations}

First of all, further research will have to carry out confirmatory factor analysis (CFA) of the QoL-AD NH and assess the proxy-report version's psychometric properties, adding validity and reliability to QoL-AD NH by comparing the self-report (during an interview) and proxy-report versions (by staff).

Researchers have long considered QoL as a secondary outcome in studies. However, QoL might, and should become, the principal outcome of interest in the coming years in nursing homes. There is a need to develop longitudinal studies and use repeated measures that are essential to assess sensitivity to change and responsiveness. However, we know the difficulty of QoL instruments in assessing significant changes in QoL measures. That is why from a clinical perspective and in agreement with Tractenberg and colleagues who compared the change in the measures of the QoL-AD 13, we think it is possible to leave the items of the QoL-AD NH as the individual QoL components to qualify the direction and quantification of this change [106]. For these researchers, accounting for change at the item level could provide more robust evidence of change in the QoL, including its improvement.

In this direction, it would be advisable to study and consider the "Response shift" phenomena. This change in response over time would consist of a change in internal standards (e.g. re-calibration), values (e.g. re-prioritisation) and reconceptualisation of what QoL can be like [107]. As a result, residents may perceive and interpret the questions put to them differently over time, depending on the course of their illness, in terms of meaning, priorities and impact on their personal life. While the response shift has been studied and taken into account with other HRQoL scales, it has never been studied in Alzheimer's disease. Consequently, other studies could try to ask the resident for each one of the items if it is "very important", "moderately important" or "not important" for them. It would also be interesting to ask the resident's opinion for each item and monitor change if it is noticed from a clinical perspective.

Also, the QoL-AD NH could constitute a baseline for QoL of residents without cognitive impairment before the disease symptoms appear [97]. Moreover, after diagnosis, the QoL-AD NH could be used to assess the resident's QoL and try to improve it, especially after a change in treatment or a non-drug intervention. Finally, staff could and should use the QoL-AD NH when drawing up the resident's life and care project in a dynamic co-construction approach involving the resident.

\section{Conclusions}

The French adaptation of the QoL-AD NH has shown globally good psychometric properties in terms of both validity and reliability for the Participant version during an interview. There is now a specific French scale to assess residents' quality of life with mild to moderate cognitive impairment. However, a CFA and further longitudinal studies are needed to evaluate sensitivity to change and responsiveness of this scale. Enabling the residents with mild to moderate cognitive impairment to assess their own QoL is already the first step and could be the bridge to build towards a person-centred approach, a resident-centred approach in France.

\section{Abbreviations}

ANESM: National agency for the evaluation and quality of social and medicosocial establishments and services; CERNI: Non-interventional Research Ethics Committee; CFA: Confirmatory Factor Analysis; EFA: Exploratory Factor Analysis; EHPAD: Établissement d'Hébergement pour Personnes Agées Dépendantes; ESEM: Exploratory Structural Equation Modeling; HRQoL: Health-related Quality of Life; ICC: Intraclass Correlation Coefficients; PA: Parallel Analysis; PROMs: 
Patient-Reported Outcome Measures; QoL: Quality of Life; QoL-AD 13: Quality of Life in Alzheimer's Disease 13 items; QoL-AD NH: Quality of Life in Alzheimer's Disease Nursing Home version; SEM: Structural Equation Modeling.

\section{Supplementary Information}

The online version contains supplementary material available at https://doi. org/10.1186/s12955-021-01853-2.

Additional file 1: Table S1. Spearman's correlation coefficient between the variables of the study $(n=174)$.

Additional file 2: Table S2. Mean, Standard deviation and Cronbach's alpha of the continuous variables of each group study.

Additional file 3: Table S3. Groups mean and standard deviation for the 15 items of the QoL-AD NH.

Additional file 4: Tables S4-S6. Standardised factor loadings, eigenvalues, intercorrelations, variance explained and Cronbach's alpha for one/ two/four-factor ESEM model.

\section{Acknowledgements}

We would like to thank the nursing home management teams who authorised us to investigate, and we warmly thank all the residents who agreed to participate in this research. We would also like to thank Rebecca Logsdon and the translators who participated in the cross-cultural adaptation of this scale: Régine Allézy, Nicole Cantisano, Maggie Wakefield and Andrea McGibney. We sincerely thank the Editors-in-Chief, the Associate Editor and the Reviewers for their constructive feedback that helped us considerably improve the quality of our manuscript. Finally, we would like to thank Pippa McKelvie-Sebileau and Jane Hentges for proofreading the final article.

\section{Authors' contributions}

CC conducted the study, analysed the results and wrote the article. VI and BQ jointly supervised the study, participated in the expert committee during the questionnaire's adaptation phase, and revised the article. All authors read and approved the final manuscript.

\section{Funding}

The research was not funded.

\section{Availability of data and materials}

QoL-AD NH (also named QoL-AD 15) is distributed by Mapi Research Trust on behalf of the copyright owner (Rebecca Logsdon): ๑ 1996, Rebecca Logsdon, PhD; University of Washington. QoL-AD NH contact information, provision and permission to use: Mapi Research Trust, Lyon, France, https://eprovide.mapitrust.org. The datasets used during the current study are available from the corresponding author on reasonable request.

\section{Declarations}

\section{Ethics approval and consent to participate}

This research was conducted in agreement with the French Code of Ethics of Psychologists. Ethical approval was obtained from the CERNI Ethics Committee of Toulouse (number 2017-064). We obtained the resident's consent to participate and, in addition, the agreement of the curator when the resident was under legal protection (See Data collection).

\section{Consent for publication}

We obtained informed consent for publication after having obtained consent to participate. Also, residents were informed that they would later have access to the study results in an accessible form that might interest them (See Data Collection).

\section{Competing interests}

The authors declare that they have no competing interests.

\section{Author details}

${ }^{1}$ CLESCO ED 326, Centre for Studies and Research in Psychopathology and Health (CERPPS), University of Toulouse Jean-Jaurès, Toulouse, France. ${ }^{2}$ INSERM BPH, UMR 1219, Team “Handicap, Activity, Cognition, Health", University of Bordeaux, Bordeaux, France.

Received: 1 June 2021 Accepted: 2 September 2021

Published online: 15 September 2021

\section{References}

1. Astier C, Noblet-Dick M, Ismer H, Fernandez C, Ebel M. P4-2 La démence, une maladie sous-diagnostiquée et sous-traitée en maison de retraite. Rev Neurol. 2005;161:128.

2. Dartigues JF, Helmer C, Letenneur L, Péres K, Amieva H, Auriacombe S, et al. Paquid 2012: illustration and overview. Geriatr Psychol Neuropsychiatr Vieil. 2012;10:325-31.

3. Villeneuve R, Meillon C, Bergua V, Tabue-Teguo M, Amieva H. Influence of pre-admission factors on quality of life and adaptation in nursing home residents with dementia: the QOL-EHPAD study protocol. BMC Geriatr. 2020;20:92.

4. KPMG. Observatoire des EHPAD 2014 [Internet]. Paris: KPMG; 2014. [cited 2021 May 15]. Available from: https://www.silvereco.fr/wp-conte nt/uploads/2014/05/Observatoire-EHPAD-2014.pdf.

5. Haute Autorité de Santé. Programme qualité de vie en ehpad. SaintDenis, France: Haute Autorité de Santé; 2012.

6. Ready RE, Ott BR, Grace J. Patient versus informant perspectives of quality of life in mild cognitive impairment and Alzheimer's disease. Int J Geriatr Psychiatry. 2004;19:256-65.

7. Smith SC, Murray J, Banerjee S, Foley B, Cook JC, Lamping DL, et al. What constitutes health-related quality of life in dementia? Development of a conceptual framework for people with dementia and their carers. Int J Geriatr Psychiatry. 2005;20:889-95.

8. Walker A, Lowenstein A. European perspectives on quality of life in old age. Eur J Ageing. 2009;6:61-6.

9. Lawton MP. Environment and other determinants of well-being in older people. Gerontologist. 1983;23:349-57.

10. Aspden T, Bradshaw SA, Playford ED, Riazi A. Quality-of-life measures for use within care homes: a systematic review of their measurement properties. Age Ageing. 2014;43:596-603.

11. Crespo M, Bernaldo de Quirós M, Gómez MM, Hornillos C. Quality of life of nursing home residents with dementia: a comparison of perspectives of residents, family, and staff. Gerontologist. 2012;52:56-65.

12. Beer C, Flicker L, Horner B, Bretland N, Scherer S, Lautenschlager NT, et al. Factors associated with self and informant ratings of the quality of life of people with dementia living in care facilities: a cross sectional study. PLOS ONE. 2010;5:e15621.

13. Edelman P, Fulton BR, Kuhn D, Chang CH. A comparison of three methods of measuring dementia-specific quality of life: perspectives of residents, staff, and observers. Gerontologist. 2005;45:27-36.

14. Sloane PD, Zimmerman S, Williams CS, Reed PS, Gill KS, Preisser JS. Evaluating the quality of life of long-term care residents with dementia. Gerontologist. 2005:45:37-49.

15. Römhild J, Fleischer S, Meyer G, Stephan A, Zwakhalen S, Leino-Kilpi H, et al. Inter-rater agreement of the Quality of Life-Alzheimer's Disease (QoL-AD) self-rating and proxy rating scale: secondary analysis of RightTimePlaceCare data. Health Qual Life Outcomes. 2018;16:131.

16. Brod M, Stewart AL, Sands L, Walton P. Conceptualization and measurement of quality of life in dementia: the dementia quality of life instrument (DQoL). Gerontologist. 1999;39:25-35.

17. Trigg R, Jones RW, Skevington SM. Can people with mild to moderate dementia provide reliable answers about their quality of life? Age Ageing. 2007;36:663-9.

18. Arlt S, Hornung J, Eichenlaub M, Jahn H, Bullinger M, Petersen C. The patient with dementia, the caregiver and the doctor: cognition, depression and quality of life from three perspectives. Int J Geriatr Psychiatry. 2008;23:604-10.

19. Thorgrimsen L, Selwood A, Spector A, Royan L, de Madariaga LM, Woods RT, et al. Whose quality of life is it anyway? The validity and 
reliability of the Quality of Life-Alzheimer's Disease (QoL-AD) scale. Alzheimer Dis Assoc Disord. 2003;17:201-8.

20. Hoe J, Katona C, Roch B, Livingston G. Use of the QOL-AD for measuring quality of life in people with severe dementia-the LASER-AD study. Age Ageing. 2005;34:130-5.

21. Hoe J, Hancock G, Livingston G, Orrell M. Quality of life of people with dementia in residential care homes. Br J Psychiatry. 2006;188:460-4.

22. Hughes $\sqcup$, Farina N, Page TE, Tabet N, Banerjee S. Psychometric properties and feasibility of use of dementia specific quality of life instruments for use in care settings: a systematic review. Int Psychogeriatr. 2019. https://doi.org/10.1017/s1041610218002259.

23. Logsdon RG, Gibbons LE, McCurry SM, Teri L. Quality of life in Alzheimer's disease: patient and caregiver reports. J Ment Health Aging. 1999;5:21-32.

24. Bowling A, Rowe G, Adams S, Sands P, Samsi K, Crane M, et al. Quality of life in dementia: a systematically conducted narrative review of dementia-specific measurement scales. Aging Ment Health. 2015;19:13-31.

25. Martyr A, Nelis SM, Quinn C, Wu YT, Lamont RA, Henderson C, et al. Living well with dementia: a systematic review and correlational metaanalysis of factors associated with quality of life, well-being and life satisfaction in people with dementia. Psychol Med. 2018;48:2130-9.

26. Bárrios H, Verdelho A, Narciso S, Gonçalves-Pereira M, Logsdon R, de Mendonça A. Quality of life in patients with cognitive impairment: validation of the Quality of Life-Alzheimer's Disease scale in Portugal. Int Psychogeriatr. 2013;25:1085-96.

27. Gómez-Gallego M, Gómez-Amor J, Gómez-García J.Validation of the Spanish version of the QoL-AD Scale in Alzheimer disease patients, their carers, and health professionals. Neurologia. 2012;27:4-10.

28. Bianchetti A, Cornali C, Ranieri P, Trabucchi M. Quality of life in patients with mild dementia. Validation of the Italian version of the quality of life Alzheimer's disease (QoL-AD) scale. J Gerontol Geriatr. 2017:65:137-43.

29. Stypa V, Haussermann P, Fleiner T, Neumann S. Validity and reliability of the German Quality of Life-Alzheimer's Disease (QoL-AD) self-report scale. J Alzheimers Dis. 2020;77:581-90.

30. Kisvetrová H, Školoudík D, Herzig R, Vališ M, Jurašková B, Krulová P, et al. Psychometric validation of the Czech version of the quality of life-Alzheimer's disease scale in patients with early-stage dementia. Dement Geriatr Cogn Disord. 2018:46:109-18.

31. Wolak A, Novella JL, Drame M, Guillemin F, Di Pollina L, Ankri J, et al. Transcultural adaptation and psychometric validation of a Frenchlanguage version of the QoL-AD. Aging Ment Health. 2009;13:593-600.

32. Moyle W, Gracia N, Murfield JE, Griffiths SG, Venturato L. Assessing quality of life of older people with dementia in long-term care: a comparison of two self-report measures. J Clin Nurs. 2012;21:1632-40.

33. Travers $C$. Increasing enjoyable activities to treat depression in nursing home residents with dementia: a pilot study. Dementia (London). 2017;16:204-18.

34. Beer C, Bosboom P, Almeida OP, Flicker L. Rating the quality of life of people with dementia living in residential care facilities in routine research practice. Age Ageing. 2009;38:343-6.

35. Dichter MN, Wolschon E-M, Schwab CGG, Meyer G, Köpke S. Item distribution and inter-rater reliability of the German version of the Quality of Life in Alzheimer's Disease scale (QoL-AD) proxy for people with dementia living in nursing homes. BMC Geriatr. 2018;18:145.

36. Winzelberg GS, Williams CS, Preisser JS, Zimmerman S, Sloane PD. Factors associated with nursing assistant quality-of-life ratings for residents with dementia in long-term care facilities. Gerontologist. 2005:45:106-14

37. Dichter MN, Wolschon EM, Meyer G, Köpke S. Cross-cultural adaptation of the German version of the Quality of Life in Alzheimer's Disease scale-Nursing Home version (QoL-AD NH). Int Psychogeriatr. 2016;28:1399-400.

38. Dichter MN, Schwab CG, Meyer G, Bartholomeyczik S, Halek M. Linguistic validation and reliability properties are weak investigated of most dementia-specific quality of life measurements-a systematic review. J Clin Epidemiol. 2016;70:233-45.

39. Nguyen KH, Mulhern B, Kularatna S, Byrnes J, Moyle W, Comans T. Developing a dementia-specific health state classification system for a new preference-based instrument AD-5D. Health Qual Life Outcomes. 2017;15:21
40. Jalenques I, Rondepierre F, Rachez C, Lauron S, Guiguet-Auclair C. Health-related quality of life among community-dwelling people aged 80 years and over: a cross-sectional study in France. Health Qual Life Outcomes. 2020;18:126.

41. Beaton DE, Bombardier C, Guillemin F, Ferraz MB. Guidelines for the process of cross-cultural adaptation of self-report measures. Spine (Phila Pa 1976). 2000;25:3186-91

42. Kalafat M, Hugonot-Diener L, Poitrenaud J. French standardization and range for the GRECO version of the 'Mini Mental State' (MMS) version Greco. Rev Neuropsychol. 2003;13:209-36.

43. Terwee CB, Bot SD, de Boer MR, van der Windt DA, Knol DL, Dekker J, et al. Quality criteria were proposed for measurement properties of health status questionnaires. J Clin Epidemiol. 2007;60:34-42.

44. Folstein MF, Folstein SE, McHugh PR. "Mini-mental state". A practical method for grading the cognitive state of patients for the clinician. J Psychiatr Res. 1975;12:189-98.

45. Monroe T, Carter M. Using the Folstein Mini Mental State Exam (MMSE) to explore methodological issues in cognitive aging research. Eur J Ageing. 2012;9:265-74.

46. Derouesne C, Poitreneau J, Hugonot L, Kalafat M, Dubois B, Laurent B. Mini-Mental State Examination:a useful method for the evaluation of the cognitive status of patients by the clinician. Consensual French version Presse Med. 1999;28:1141-8.

47. Schwebel G, Dramé M, Jolly D, Boyer F, Morrone I, Di Pollina L, et al. Validation psychométrique du questionnaire Dementia Quality of Life (DQoL) en langue française. Revue Gériatr. 2010;35:93-100.

48. Santana-Berlanga NDR, Porcel-Gálvez AM, Botello-Hermosa A, Barrientos-Trigo S. Instruments to measure quality of life in institutionalised older adults: systematic review. Geriatr Nurs. 2020;41:445-62.

49. Yesavage JA, Brink TL, Rose TL, Lum O, Huang V, Adey M, et al. Development and validation of a geriatric depression screening scale: a preliminary report. J Psychiatr Res. 1982;17:37-49.

50. Yesavage JA, Sheikh J. Geriatric Depression Scale (GDS): recent evidence and development of a shorter version. Clin Gerontol. 1986:5:165-73.

51. Bourque P, Blanchard L, Vézina J. Étude psychométrique de l'Échelle de dépression gériatrique. Can J Aging. 2010;9:348-55.

52. Conradsson M, Rosendahl E, Littbrand H, Gustafson Y, Olofsson B, Lövheim H. Usefulness of the geriatric depression scale 15-item version among very old people with and without cognitive impairment. Aging Ment Health. 2013;17:638-45.

53. Fayers PM, Machin D. Quality of life: the assessment, analysis, and reporting of patient-reported outcomes. Hoboken: Wiley; 2016.

54. Hair JF, Hult GTM, Ringle CM, Sarstedt M. A primer on partial least squares structural equation modeling (PLS-SEM). Thousand Oaks: Sage; 2017.

55. Kline R. Principles and practice of structural equation modeling. New York: Guilford Publications; 2010.

56. Tabachnick BG, Fidell LS. Using multivariate statistics. Boston: Pearson/ Allyn \& Bacon; 2007.

57. Asparouhov T, Muthén B. Exploratory structural equation modeling. Struct Equ Model Multidiscip J. 2009;16:397-438.

58. Marsh HW, Morin AJ, Parker PD, Kaur G. Exploratory structural equation modeling: an integration of the best features of exploratory and confirmatory factor analysis. Annu Rev Clin Psychol. 2014;10:85-110.

59. Morin AJS, Maïano C. Cross-validation of the short form of the physical self-inventory (PSI-S) using exploratory structural equation modeling (ESEM). Psychol Sport Exerc. 2011;12:540-54.

60. Maïano C, Morin AJ, Lanfranchi MC, Therme P. The eating attitudes test26 revisited using exploratory structural equation modeling. J Abnorm Child Psychol. 2013;41:775-88.

61. Henson RK, Roberts JK. Use of exploratory factor analysis in published research: common errors and some comment on improved practice. Educ Psychol Meas. 2006;66:393-416.

62. Marsh HW, Muthén B, Asparouhov T, Lüdtke O, Robitzsch A, Morin AJS, et al. Exploratory structural equation modeling, integrating CFA and EFA: application to students' evaluations of University teaching. Struct Equ Model Multidiscip J. 2009;16:439-76.

63. Guàrdia-Olmos J, Peró-Cebollero M, Benítez-Borrego S, Fox J. Using sem library in $r$ software to analyze exploratory structural equation models. In: Proceedings of the 59th ISI World Statistics Congress; 2013 
Aug 25-30; Hong Kong, China. The Hague: The International Statistical Institute; 2013 [cited 2021 May 17]. p. 4600-5. Available from: https:// 2013.isiproceedings.org/Files/CPS105-P6-S.pdf.

64. Hu Lt, Bentler PM. Cutoff criteria for fit indexes in covariance structure analysis: conventional criteria versus new alternatives. Struct Equ Model Multidiscip J. 1999;6:1-55.

65. Cheung GW, Rensvold RB. Evaluating goodness-of-fit indexes for testing measurement invariance. Struct Equ Model Multidiscip J. 2002;9:233-55.

66. Portney LG, Watkins MP. Foundations of clinical research: applications to practice. Upper Saddle River: Pearson/Prentice Hall; 2009.

67. Netemeyer RG, Bearden WO, Sharma S. Scaling procedures: issues and applications. Thousand Oaks: Sage Publications; 2003.

68. Kline RB. Principles and practice of structural equation modeling. New York: The Guilford Press; 2016.

69. Rodriguez A, Reise SP, Haviland MG. Evaluating bifactor models: calculating and interpreting statistical indices. Psychol Methods. 2016;21:137-50.

70. McDonald RP. Test theory: a unified treatment. London: Psychology Press; 2013.

71. Watkins MW. The reliability of multidimensional neuropsychological measures: from alpha to omega. Clin Neuropsychol. 2017;31:1113-26.

72. Hayes AF, Coutts JJ. Use omega rather than Cronbach's alpha for estimating reliability. But.... Commun Methods Meas. 2020;14:1-24.

73. Flora DB. Your coefficient alpha is probably wrong, but which coefficient omega is right? A tutorial on using $\mathrm{R}$ to obtain better reliability estimates. Adv Methods Pract Psychol Sci. 2020;3:484-501.

74. Reise SP. The rediscovery of bifactor measurement models. Multivar Behav Res. 2012;47:667-96.

75. Fornell C, Larcker DF. Evaluating structural equation models with unobservable variables and measurement error. J Mark Res. 1981;18:39-50.

76. Reise SP, Bonifay WE, Haviland MG. Scoring and modeling psychological measures in the presence of multidimensionality. J Pers Assess. 2013:95:129-40

77. Koo TK, Li MY. A guideline of selecting and reporting intraclass correlation coefficients for reliability research. J Chiropr Med. 2016:15:155-63.

78. R Core Team. R: a language and environment for statistical computing Vienna: R Foundation for Statistical Computing; 2020.

79. RStudio Team. RStudio: integrated development for R. RStudio. Boston: RStudio Team; 2021

80. Farquhar M. Elderly people's definitions of quality of life. Soc Sci Med. 1995:41:1439-46.

81. Collins JH. The person-centered way: revolutionizing quality of life in long-term care. Place of publication not identified. South Carolina, US BookSurge Publishing; 2009

82. Cahill S, Diaz-Ponce AM. "I hate having nobody here. I'd like to know where they all are": can qualitative research detect differences in quality of life among nursing home residents with different levels of cognitive impairment? Aging Ment Health. 2011;15:562-72.

83. Zeisel J. I'm still here: a new philosophy of Alzheimer's care. New York: Avery; 2009.

84. den Ouden M, Bleijlevens MH, Meijers JM, Zwakhalen SM, Braun SM Tan FE, et al. Daily (in)activities of nursing home residents in their wards: an observation study. J Am Med Dir Assoc. 2015;16:963-8.

85. Kitwood T. Dementia reconsidered: the person comes first. Philadel phia: Open University Press; 1997

86. Camp CJ. Hiding the stranger in the mirror: a detective's manual for solving problems associated with Alzheimer's Disease and related distorders. Solon: Center for Applied Research in Dementia; 2008.

87. Roberts G, Morley C, Walters W, Malta S, Doyle C. Caring for people with dementia in residential aged care: successes with a composite personcentered care model featuring Montessori-based activities. Geriatr Nurs. 2015;36:106-10

88. Leplège A. Les mesures de la qualité de vie. Paris: Presses Universitaires de France; 1999.

89. Aaronson NK, Ahmedzai S, Bergman B, Bullinger M, Cull A, Duez NJ, et al. The European organization for research and treatment of cancer QLQ-C30: a quality-of-life instrument for use in international clinical trials in oncology. J Natl Cancer Inst. 1993;85:365-76.
90. Ware JE Jr Sherbourne CD. The MOS 36-item short-form health survey (SF-36). I. Conceptual framework and item selection. Med Care. 1992:30:473-83.

91. Souto T, Ramires A, Leite $\hat{A}$, Santos V, Santo R. Health perception: validation of a scale for the portuguese population. Temas em Psicol. 2018;26:2167-83.

92. Schenk L, Meyer R, Behr A, Kuhlmey A, Holzhausen M. Quality of life in nursing homes: results of a qualitative resident survey. Qual Life Res. 2013:22:2929-38.

93. Conde-Sala JL, Reñé-Ramírez R, Turró-Garriga O, Gascón-Bayarri J, Juncadella-Puig M, Moreno-Cordón L, et al. Clinical differences in patients with Alzheimer's disease according to the presence or absence of anosognosia: implications for perceived quality of life. J Alzheimers Dis. 2013;33:1105-16.

94. Portellano-Ortiz C, Turró-Garriga O, Gascón-Bayarri J, Piñán-Hernández S, Moreno-Cordón L, Viñas-Díez V, et al. The influence of anosognosia and depression on the perceived quality of life of patients with Alzheimer's disease: a 12 months follow-up. Rev Neurol. 2014;59:193-204.

95. Conde-Sala JL, Turró-Garriga O, Piñán-Hernández S, Portellano-Ortiz C, Viñas-Diez V, Gascón-Bayarri J, et al. Effects of anosognosia and neuropsychiatric symptoms on the quality of life of patients with Alzheimer's disease: a 24-month follow-up study. Int J Geriatr Psychiatry. 2016:31:109-19.

96. Gómez-Gallego M, Gomez-García J, Ato-García M. Confirmatory factor analysis of the quality of life in Alzheimer's disease scale in patients with Alzheimer's disease. Exp Aging Res. 2014;40:266-79.

97. Revell AJ, Caskie GI, Willis SL, Schaie KW. Factor structure and invariance of the Quality of Life in Alzheimer's Disease (QoL-AD) scale. Exp Aging Res. 2009;35:250-67.

98. Yamada T, Nakaaki S, Sato J, Sato H, Shikimoto R, Furukawa TA, et al. Factor structure of the Japanese version of the Quality of Life in Alzheimer's Disease Scale (QOL-AD). Psychogeriatrics. 2020;20:79-86.

99. Costello AB, Osborne J. Best practices in exploratory factor analysis: four recommendations for getting the most from your analysis. Pract Assess Res Eval. 2005;10:1-9.

100. Brazier JE, Rowen D, Mavranezouli I, Tsuchiya A, Young T, Yang Y, et al. Developing and testing methods for deriving preference-based measures of health from condition-specific measures (and other patientbased measures of outcome). Health Technol Assess. 2012;16:1-114

101. Stamper CL, Masterson SS. Insider or outsider? How employee perceptions of insider status affect their work behavior. J Organ Behav. 2002:23:875-94.

102. Wolak-Thierry A, Novella JL, Barbe C, Morrone I, Mahmoudi R, Jolly D. Comparison of QoL-AD and DQoL in elderly with Alzheimer's disease. Aging Ment Health. 2015;19:274-8.

103. Ayton DR, Gardam ML, Pritchard EK, Ruseckaite R, Ryan J, Robinson SJ, et al. Patient-reported outcome measures to inform care of people with dementia-A systematic scoping review. Gerontologist. 2021:61:e185-94.

104. Rosas-Carrasco O, Torres-Arreola Ldel P, Guerra-Silla MDG, Torres-Castro S, Gutierrez-Robledo LM. Validation of the Quality of Life in Alzheimer's Disease (QOL-AD) scale in Mexican patients with Alzheimer, vascular and mixed-type dementia. Rev Neurol. 2010;51:72-80.

105. Fokkema M, Greiff S. How performing PCA and CFA on the same data equals trouble - overfitting in the assessment of internal structure and some editorial thoughts on it. Eur J Psychol Assess. 2017;33:399-402.

106. Tractenberg R, Yumoto F, Aisen P. Detecting when "quality of life" has been "enhanced": estimating change in quality of life ratings. Open J Philos. 2013;03:24-31.

107. Sprangers MA, Schwartz CE. Integrating response shift into healthrelated quality of life research: a theoretical model. Soc Sci Med. 1999:48:1507-15.

\section{Publisher's Note}

Springer Nature remains neutral with regard to jurisdictional claims in published maps and institutional affiliations. 